\title{
Cooperative Peer-to-Peer Streaming: An Evolutionary Game-Theoretic Approach
}

\author{
Yan Chen, Student Member, IEEE, Beibei Wang, Student Member, IEEE, W. Sabrina Lin, Member, IEEE, \\ Yongle Wu, Student Member, IEEE, and K. J. Ray Liu, Fellow, IEEE
}

\begin{abstract}
While peer-to-peer (P2P) video streaming systems have achieved promising results, they introduce a large number of unnecessary traverse links, which consequently leads to substantial network inefficiency. To address this problem and achieve better streaming performance, we propose to enable cooperation among "group peers," which are geographically neighboring peers with large intra-group upload and download bandwidths. Considering the peers' selfish nature, we formulate the cooperative streaming problem as an evolutionary game and derive, for every peer, the evolutionarily stable strategy (ESS), which is the stable Nash equilibrium and no one will deviate from. Moreover, we propose a simple and distributed learning algorithm for the peers to converge to the ESSs. With the proposed algorithm, each peer decides whether to be an agent who downloads data from the peers outside the group or a freerider who downloads data from the agents by simply tossing a coin, where the probability of being a head for the coin is learned from the peer's own past payoff history. Simulation results show that the strategy of a peer converges to the ESS. Compared to the traditional non-cooperative $\mathrm{P} 2 \mathrm{P}$ schemes, the proposed cooperative scheme achieves much better performance in terms of social welfare, probability of real-time streaming, and video quality (source rate).
\end{abstract}

Index Terms-Cooperative streaming, distributed learning, evolutionary, game theory, peer-to-peer (P2P), replicator dynamics.

\section{INTRODUCTION}

W ITH THE RAPID development of signal processing, communication, and networking technologies, videoover-IP applications become more and more popular and have attracted millions of users over the Internet [1], [2]. One simple solution to video streaming over Internet is the clientserver service model [3], [4], where the video is streamed directly from a server to clients. However, with the clientserver service model, the upload bandwidth of the server grows proportionally with the number of clients [5], which makes the large-scale video streaming impractical.

To reduce the workload of the server, peer-to-peer (P2P) service model is proposed [6], [7], where a peer not only acts

Manuscript received September 17, 2009; revised April 20, 2010; accepted June 16, 2010. Date of publication September 20, 2010; date of current version October 8, 2010. This paper was recommended by Associate Editor I. Ahmad.

The authors are with the Department of Electrical and Computer Engineering, University of Maryland, College Park, MD 20742 USA (email: yan@umd.edu; bebewang@umd.edu; wylin@umd.edu; wuyl@umd.edu; kjrliu@umd.edu).

Color versions of one or more of the figures in this paper are available online at http://ieeexplore.ieee.org.

Digital Object Identifier 10.1109/TCSVT.2010.2077490 as a client to download data from the network, but also acts as a server to upload data for the other peers in the network. The upload bandwidth of the peers reduces the workload placed on the server dramatically, which makes large-scale video streaming possible. Recently, several industrial large-scale P2P video streaming systems have been developed, including Coolstreaming [7], PPLive [8], PPStream [9], UUSee [10], and Sopcast [11]. Studies show that these systems can support hundreds of thousands of users simultaneously [12].

While P2P video streaming systems have achieved promising results, they have several drawbacks. First, there are a large number of unnecessary traverse links within a provider's network. As observed in [13], each P2P bit on the Verizon network traverses 1000 miles and takes 5.5 metro-hops on average. Second, there is a huge number of cross Internet service provider traffic. The studies in [14] and [15] showed that $50 \%-90 \%$ of the existing local pieces in active peers are downloaded externally. Third, the differences in playback time among peers can be as high as $140 \mathrm{~s}$ [12], and the lag can be greater if the source rate is higher. Fourth, most of the current P2P systems assume that all peers are willing to contribute their resources. However, this assumption may not be true since the P2P systems are self-organizing networks and the peers are selfish by nature [16], [17]. Note that the selfish peers will act as free-riders if being free-riders can improve their utilities.

In the literature, many approaches have been proposed to overcome these drawbacks. Karagiannis et al. [14] and Madhyastha et al. [18] proposed to use locality-aware P2P schemes to reduce the unnecessary traverse links within and cross ISPs and thus reduce the download time. Purandare and Guha [19] proposed an alliance based peering scheme to reduce the playback time lag and improve the quality of service. Xie et al. [13] proposed a P4P architecture that allows cooperative traffic control between applications and network providers. To stimulate selfish peers to contribute their resources, payment mechanisms [20], [21] and reputation schemes [22], [23] are proposed, where peers pay points to receive data and earn points by forwarding data to others. However, such payment or reputation based mechanisms often demand a centralized architecture and thus hinder their scalability.

Game theory is a mathematical tool that analyzes the strategic interactions among multiple decision makers. Recently, it draws great attention in cognitive networking [24], multimedia social networking [25], and is being applied to 
many multimedia signal processing problems such as video coding [26] and multimedia communications [27]. In P2P networks, peers make intelligent decisions on their strategies of requesting and forwarding packets based on their needs and other peers' actions. Moreover, since peers are rational and thus naturally selfish, they have no incentive to contribute their resources for other peers. Therefore, it is natural to study the intelligent behaviors and interactions of selfish peers in P2P networks from a game-theoretic perspective [16], [28]. Using a mental cost to describe the level of the peer's altruism, the authors in [16] presented a game-theoretical model to analyze nodes' behaviors and the influence of incentive mechanism. In [28], a game-theoretic framework is proposed for designing distributed, cheat-proof, and attack-resistant cooperation stimulation strategies for P2P live streaming social networks.

Most of the existing schemes treat every peer as an independent individual. However, in reality, every peer can have a large number of geographically neighboring peers with large intra-group upload and download bandwidths, e.g., the peers in the same lab, building, or campus. In this paper, we name those geographically neighboring peers with large intra-group upload and download bandwidths as group peers. To reduce the unnecessary traverse links and improve network efficiency, instead of considering each peer's strategy independently, we investigate possible cooperation among the group peers. Moreover, since peers are naturally selfish, they will act as free-riders if doing so can improve their utilities. In such a case, full cooperation cannot be guaranteed. Instead, to achieve better payoff, rational peers will adjust their degree of cooperation by learning from their payoff history. Therefore, a key question to answer is how a group of selfish peers should cooperate with each other to achieve better streaming performance.

The main contributions of this paper are summarized as follows.

1) We propose a cooperative streaming scheme to enable cooperation among group peers to achieve better streaming performance.

2) In the proposed scheme, we define the utility function of a peer by taking into account the possibility of real-time streaming and the cost of acting as a server to upload data for the other peers.

3) Due to their selfish nature, peers tend to act as free riders to improve their own utilities. Moreover, the peers may take out-of-equilibrium strategies due to the uncertainty of the strategies of the other peers. Therefore, a robust Nash equilibrium (NE) solution is desired for every peer. In this paper, we formulate the cooperative streaming problem as an evolutionary game and derive the evolutionarily stable strategy (ESS) for every peer, which is the desired stable NE.

4) To stimulate cooperation, the cooperative streaming scheme should be simple since peers may not be willing to join the cooperative streaming if the protocol is complicated. The proposed cooperative streaming scheme is very simple. Each peer tosses a coin to decide whether to be an agent or a free rider. If the outcome is head, the

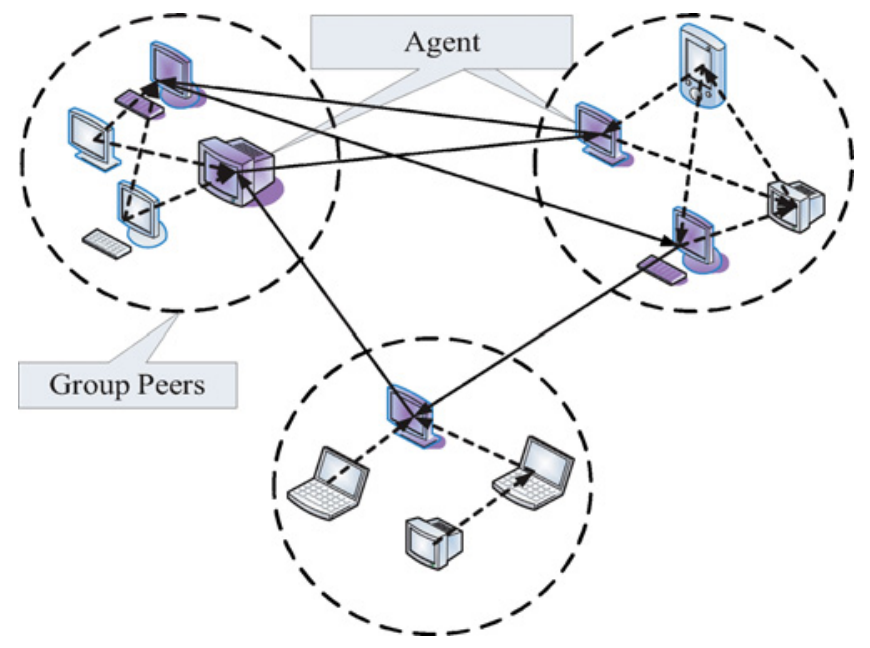

Fig. 1. Cooperative streaming example.

peer acts as an agent to download data from the peers outside the group. Otherwise, the peer acts as a free-rider to download data from the agents. And the probability of being a head for the coin is learned from the peer's own past payoff history.

5) Due to the highly dynamic behaviors of the peers, i.e., the peers may join or leave the P2P network at any time, the cooperative streaming scheme should be distributed. We propose a distributed algorithm for every peer to approach the ESS by learning from the peer's own past payoff history.

The rest of this paper is organized as follows. In Section II, we describe the system model and the utility function. Then, we show in details how to select agents in a homogeneous group in Section III. We extend the analysis to the heterogeneous case in Section IV. In Section V, we propose a distributed learning algorithm for ESS. Finally, we show the simulation results in Section VI and draw conclusions in Section VII.

\section{SYSTEM MODEL AND UTILITY FUNCTION}

\section{A. System Model}

As shown in Fig. 1, there is a set of group peers ${ }^{1}$ (three in this example) who want to view a real-time video streaming simultaneously. Within a group, every peer can choose either to be an agent or a normal peer. If the peer serves as an agent, he/she not only needs to act as a client to download video data from the agents in other groups, but also needs to act as a server to upload video streams for both the agents in other groups and the peers in the same group. However, if the peer chooses not to be an agent, he/she only needs to download/upload data from/to the peers in the same group. We assume that the upload and download bandwidth within the group is larger than those cross groups. In such a case,

\footnotetext{
${ }^{1}$ How to group the peers itself is an interesting problem. However, in this paper, we assume that the peers have already been grouped and mainly focus on how the group peers cooperate with each other to achieve better streaming performance.
} 
peers tend to be a normal peer due to the selfish nature. Nevertheless, the normal peers, on the other hand, take a risk of receiving degraded streaming performance since there may not be sufficient agents to download data from other groups. In order to achieve good streaming performance through cooperation, a question need to be addressed: given a group of peers, which peers should serve as agents?

\section{B. Utility Functions}

In a $\mathrm{P} 2 \mathrm{P}$ network, a peer not only acts as a client to download video data from the other peers but also acts as a server to upload video data for the other peers. Therefore, while a peer can benefit from downloading data from the other peers, he/she also incurs a cost in uploading data for the other peers, where the cost can be resource spending on uploading data, e.g., bandwidth, buffer size.

Given the group peers, $u_{1}, u_{2}, \ldots, u_{N}$, we assume that $k$ peers are willing to serve as agents to download multimedia data from the peers outside the group. Let the download rate be the transmission speed between an agent and a corresponding peer outside the group. If we denote that the download rates of the $k$ agents are $r_{1}, r_{2}, \ldots, r_{k}$, then the total download rate of the group peers is given by

$$
y_{k}=\sum_{i=1}^{k} r_{i} .
$$

Since the agents randomly and independently select peers outside the group for downloading data, the download rate $r_{i} \mathrm{~s}$ are random variables. According to [29], the cumulative distribution function of a peer's download bandwidth can be modelled as a linear function, which means that the PDF of a peer's download bandwidth can be modelled as a uniform distribution, i.e., $r_{i}$ s are uniformly distributed.

To provide more insight into the cooperative streaming problem, we first consider a simple scenario without buffering. Then, we extend our discussion to the case when there is buffering effect in Section VI. For the scenario without buffering, if the total download rate $y_{k}$ is not smaller than the source rate $r$, then the group peers can have a real-time streaming, and all the group peers can obtain a certain gain $G$. Otherwise, there will be some delay, and in this case we assume the gain is zero. Therefore, given the total download rate $y_{k}$ and the source rate $r$, if peer $u_{i}$ chooses to be an agent, then the utility function of $u_{i}$ is given by

$$
U_{A, i}(k)=\operatorname{Pr}\left(y_{k} \geq r\right) G-C_{i} \quad \forall k \in[1, N]
$$

where $C_{i}$ is the cost of $u_{i}$ when he/she serves as an agent, and $\operatorname{Pr}\left(y_{k} \geq r\right)$ is the probability of achieving a real-time streaming which can be computed according to Theorem 1 .

Since the upload and download bandwidths within the group are large, the cost of uploading data to the other peers within the group can be negligible. In such a case, if peer $u_{i}$ chooses not to be an agent, then there is no cost for $u_{i}$ and the utility function becomes

$$
U_{N, i}(k)= \begin{cases}\operatorname{Pr}\left(y_{k} \geq r\right) G & \text { if } k \in[1, N-1] \\ 0 & \text { if } k=0 .\end{cases}
$$

Theorem 1: If $r_{1}, r_{2}, \ldots, r_{k}$ are i.i.d. uniformly distributed within $\left[r^{L}, r^{U}\right]$, then $\operatorname{Pr}\left(y_{k} \geq r\right)$ is given by

$$
\begin{aligned}
& \operatorname{Pr}\left(y_{k} \geq r\right) \\
= & \frac{1}{2 k !} \sum_{l=0}^{k}(-1)^{l}\left(\begin{array}{l}
k \\
l
\end{array}\right)\left[(k-l)^{k}-\operatorname{sgn}(\hat{r}-l)(\hat{r}-l)^{k}\right]
\end{aligned}
$$

and when $k$ is sufficiently large, $\operatorname{Pr}\left(y_{k} \geq r\right)$ can be approximated as

$$
\operatorname{Pr}\left(y_{k} \geq r\right) \approx Q\left(\frac{\hat{r}-\frac{k}{2}}{\sqrt{\frac{k}{12}}}\right)
$$

where $\hat{r}=\frac{r-k r^{L}}{r^{U}-r^{L}}$ and $Q(x)$ is the Gaussian tail function $\int_{x}^{\infty} \frac{1}{\sqrt{2 \pi}} \exp ^{-\frac{x^{2}}{2}} d x$.

Proof: See Appendix A.

\section{Agents Selection Within A Homogeneous GROUP}

In the previous section, we have discussed the system model and the peer's utility function. To optimize the streaming performance, proper peers should serve as agents to download data from the peers outside the group. In this section, we will discuss how to select agents within a homogeneous group where the cost of all peers serving as an agent is assumed to be the same.

\section{A. Centralized Agent Selection}

If there is a central controller who can choose which peers should act as agents, then a straightforward criterion of selecting proper agents is to maximize the social welfare, which is the sum of all peers' utilities.

Let $C_{i}=C$ be the cost of a peer serving as an agent in a homogeneous group. Then the social welfare of an $N$-peer group with $k$ agents can be calculated by

$$
S W(k)=\operatorname{Pr}\left(y_{k} \geq r\right) G N-k C .
$$

Based on (6), the agent selection problem to maximize the social welfare can be formulated as

$$
\max _{k} S W(k)=\max _{k}\left[\operatorname{Pr}\left(y_{k} \geq r\right) G N-k C\right]
$$

where $k \in\{1,2, \ldots, N\}$.

By solving (7), we can find the optimal $k^{\star}$ that maximizes the social welfare. Then, the central controller can choose $k^{\star}$ peers from the group as agents to download data from the peers outside the group based on some mechanism, e.g., the peers take turns to serve as agents. However, since peers' behaviors are highly dynamic, they may join in or leave the P2P network at any time. In such a case, the centralized approach may not be practical.

\section{B. Distributed Agent Selection}

To overcome the drawback of the centralized approach, it is possible to consider a distributed approach where each peer 
acts as an agent with probability $x$. Then, according to (2) and (3), the group's social welfare can be computed by

$$
U_{\text {total }}(x)=\sum_{i=1}^{N}\left(\begin{array}{c}
N \\
i
\end{array}\right) x^{i}(1-x)^{N-i}\left[\operatorname{Pr}\left(y_{i} \geq r\right) G N-i C\right] \text {. }
$$

The problem of finding an optimal $x$ to maximize the social welfare can be formulated as

$$
\begin{aligned}
& \max _{x} \sum_{i=1}^{N}\left(\begin{array}{c}
N \\
i
\end{array}\right) x^{i}(1-x)^{N-i}\left[\operatorname{Pr}\left(y_{i} \geq r\right) G N-i C\right] \\
& \text { s.t. } \quad 0 \leq x \leq 1 .
\end{aligned}
$$

However, since peers are selfish by nature, they are not as cooperative as a system designer/controller desires. By solving (9), we can find the optimal $x^{\star}$ that maximizes the social welfare, but $x^{\star}$ cannot maximize each peer's own utility. Therefore, the social welfare maximizer $x^{\star}$ is not attainable when peers are selfish. Moreover, the solution to the optimization problem shown in (9) is not stable since any perturbation will lead to a new solution.

\section{Evolutionary Cooperative Streaming Game}

In order to provide a robust equilibrium strategy for the selfish peers, we adopt the concept of ESS [30], [31], which is defined as follows.

Definition 1: A strategy $a^{\star}$ is an ESS if and only if, $\forall a \neq a^{\star}$, $a^{\star}$ satisfies:

1) equilibrium condition: $U_{i}\left(a, a^{\star}\right) \leq U_{i}\left(a^{\star}, a^{\star}\right)$;

2) stability condition: if $U_{i}\left(a, a^{\star}\right)=U_{i}\left(a^{\star}, a^{\star}\right), U_{i}(a, a)<$ $U_{i}\left(a^{\star}, a\right)$

where $U_{i}\left(a_{1}, a_{2}\right)$ is the utility of player $i$ when he/she uses strategy $a_{1}$ and another player uses strategy $a_{2}$.

Since all peers are selfish, they will cheat if cheating can improve their payoffs, which means that all peers are uncertain of other peers' actions and utilities. In such a case, to improve their utilities, peers will try different strategies in every play and learn from the strategic interactions using the methodology of understanding-by-building. During the process, the percentage of peers using a certain pure strategy may change. Such a population evolution can be modelled by replicator dynamics. Specifically, let $x_{a}$ stand for the probability of a peer using pure strategy $a \in \mathcal{A}$, where $\mathcal{A}=\{A, N\}$ is the set of pure strategies including being an agent $(A)$ and not being an agent $(N)$. By replicator dynamics, the evolution dynamics of $x_{a}$ are given by the following differential equation:

$$
\dot{x}_{a}=\eta\left[\bar{U}\left(a, x_{-a}\right)-\bar{U}\left(x_{a}\right)\right] x_{a}
$$

where $\bar{U}\left(a, x_{-a}\right)$ is the average payoff of the peers using pure strategy $a, x_{-a}$ is the set of peers who use pure strategies other than $a, \bar{U}\left(x_{a}\right)$ is the average payoff of all peers, and $\eta$ is a positive scale factor.

From (10), we can see that if adopting pure strategy $a$ can lead to a higher payoff than the average level, the probability of a peer using $a$ will grow and the growth rate $\dot{x}_{a} / x_{a}$ is proportional to the difference between the average payoff of using strategy $a$ [i.e., $\left.\bar{U}\left(a, x_{-a}\right)\right]$ and the average payoff of all peers [i.e., $\bar{U}\left(x_{a}\right)$ ].

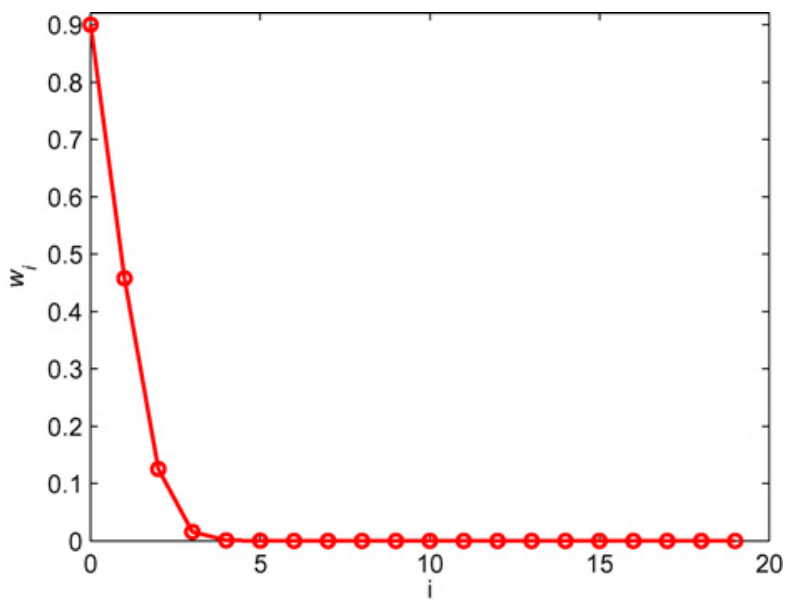

Fig. 2. Deceasing property of $w_{i}$.

\section{Analysis of the Cooperative Streaming Game}

According to (2) and (3), the average payoff of a peer if he/she choose to be an agent can be computed by

$$
\bar{U}_{A}(x)=\sum_{i=0}^{N-1}\left(\begin{array}{c}
N-1 \\
i
\end{array}\right) x^{i}(1-x)^{N-1-i}\left[\operatorname{Pr}\left(y_{i+1} \geq r\right) G-C\right]
$$

where $x$ is the probability of a peer being an agent, and $\left(\begin{array}{c}N-i \\ i\end{array}\right) x^{i}(1-x)^{N-1-i}$ is the probability that there are $i$ agents out of $N-1$ other peers.

Similarly, the average payoff of a peer if he/she chooses not to be an agent is given by

$$
\bar{U}_{N}(x)=\sum_{i=1}^{N-1}\left(\begin{array}{c}
N-1 \\
i
\end{array}\right) x^{i}(1-x)^{N-1-i} \operatorname{Pr}\left(y_{i} \geq r\right) G .
$$

According to (11) and (12), the average payoff of a peer is

$$
\bar{U}(x)=x \bar{U}_{A}(x)+(1-x) \bar{U}_{N}(x) .
$$

Substituting (13) back to (10), we have

$$
\dot{x}=\eta x(1-x)\left[\bar{U}_{A}(x)-\bar{U}_{N}(x)\right] .
$$

At equilibrium $x^{\star}$, no player will deviate from the optimal strategy, which means $\dot{x}^{\star}=0$, and we can obtain $x^{\star}=0,1$, or the solutions to $\bar{U}_{A}(x)=\bar{U}_{N}(x)$. However, since $\dot{x}^{\star}=0$ is only the necessary condition for $x^{\star}$ to be ESS, we examine the sufficient condition for each ESS candidate and draw the following conclusions with the proofs shown in Theorems 2-4.

1) $x^{\star}=0$ is an ESS only when $\operatorname{Pr}\left(y_{1} \geq r\right) G-C \leq 0$.

2) $x^{\star}=1$ is an ESS only when $\operatorname{Pr}\left(y_{N} \geq r\right) G-\operatorname{Pr}\left(y_{N-1} \geq\right.$ $r) G \geq C$.

3) Let $x^{\star}$ be the solution to $\bar{U}_{A}(x)=\bar{U}_{N}(x)$, and $x^{\star} \in(0,1)$. Then, $x^{\star}$ is an ESS.

Lemma 1: Let $f(x)=\bar{U}_{A}(x)-\bar{U}_{N}(x)$, then $f^{\prime}(x)<0, \forall x \in$ $[0,1]$. 
Proof: According to (11) and (12), we have

$$
f(x)=\sum_{i=0}^{N-1}\left(\begin{array}{c}
N-1 \\
i
\end{array}\right) x^{i}(1-x)^{N-1-i} w_{i}-C
$$

where $w_{i}=\left[\operatorname{Pr}\left(y_{i+1} \geq r\right)-\operatorname{Pr}\left(y_{i} \geq r\right)\right] G$.

1) $\forall x \in(0,1)$, by taking the derivative of $f(x)$ over $x$, we have

$$
\begin{aligned}
f^{\prime}(x) & =\sum_{i=0}^{N-1}\left(\begin{array}{c}
N-1 \\
i
\end{array}\right) x^{i-1}(1-x)^{N-2-i}[i-(N-1) x] w_{i} \\
& =\sum_{i=0}^{i_{1}}\left(\begin{array}{c}
N-1 \\
i
\end{array}\right) x^{i-1}(1-x)^{N-2-i}[i-(N-1) x] w_{i} \\
& +\sum_{i=i_{1}+1}^{N-1}\left(\begin{array}{c}
N-1 \\
i
\end{array}\right) x^{i-1}(1-x)^{N-2-i}[i-(N-1) x] w_{i}
\end{aligned}
$$

where $i_{1}$ is the integer such that $i_{1} \leq(N-1) x$ and $i_{1}+1>(N-1) x$.

Since $w_{i}$ stands for the additional gain by introducing one more agent into the $i$-agent system, as shown in Fig. 2 , it is a decreasing function in terms of $i$, which means that $w_{i} \geq w_{i_{1}}, \forall i \leq i_{1}$ and $w_{i} \leq w_{i_{1}}, \forall i>i_{1}$. Therefore, according to (16), we have

$$
\begin{aligned}
f^{\prime}(x) & <\sum_{i=0}^{i_{1}}\left(\begin{array}{c}
N-1 \\
i
\end{array}\right) x^{i-1}(1-x)^{N-2-i}[i-(N-1) x] w_{i_{1}} \\
& +\sum_{i=i_{1}+1}^{N-1}\left(\begin{array}{c}
N-1 \\
i
\end{array}\right) x^{i-1}(1-x)^{N-2-i}[i-(N-1) x] w_{i_{1}} \\
& =w_{i_{1}} \sum_{i=0}^{N-1}\left(\begin{array}{c}
N-1 \\
i
\end{array}\right) x^{i-1}(1-x)^{N-2-i}[i-(N-1) x] \\
& =w_{i_{1}} \frac{d\left[\sum_{i=0}^{N-1}\left(\begin{array}{c}
N-1 \\
i
\end{array}\right) x^{i}(1-x)^{N-1-i}\right]}{d x} \\
& =0 .
\end{aligned}
$$

Therefore, $f^{\prime}(x)<0, \forall x \in(0,1)$.

2) The derivative of $f(x)$ over $x$ at $x=0$ can be computed by

$$
\begin{aligned}
f^{\prime}(0) & =\lim _{\varepsilon \rightarrow 0} \frac{f(\varepsilon)-f(0)}{\varepsilon} \\
& =\lim _{\varepsilon \rightarrow 0} \frac{\sum_{i=0}^{N-1}\left(\begin{array}{c}
N-1 \\
i
\end{array}\right) \varepsilon^{i}(1-\varepsilon)^{N-1-i} w_{i}-w_{0}}{\varepsilon} \\
& =\lim _{\varepsilon \rightarrow 0} \frac{(1-\varepsilon)^{N-1} w_{0}-w_{0}}{\varepsilon} \\
& +\lim _{\varepsilon \rightarrow 0} \frac{(N-1) \varepsilon(1-\varepsilon)^{N-2} w_{1}}{\varepsilon} \\
& =(N-1)\left(w_{1}-w_{0}\right) \\
& <0
\end{aligned}
$$

where the last inequality comes from the fact that $w_{i}$ is a decreasing function in terms of $i$.
3) Similarly, the derivative of $f(x)$ over $x$ at $x=1$ can be computed by

$$
\begin{aligned}
f^{\prime}(1) & =\lim _{\varepsilon \rightarrow 0} \frac{f(1)-f(1-\varepsilon)}{\varepsilon} \\
& =\lim _{\varepsilon \rightarrow 0} \frac{w_{N-1}-\sum_{i=0}^{N-1}\left(\begin{array}{c}
N-1 \\
i
\end{array}\right)(1-\varepsilon)^{i} \varepsilon^{N-1-i} w_{i}}{\varepsilon} \\
& =\lim _{\varepsilon \rightarrow 0} \frac{w_{N-1}-(1-\varepsilon)^{N-1} w_{N-1}}{\varepsilon} \\
& +\lim _{\varepsilon \rightarrow 0} \frac{-(N-1)(1-\varepsilon)^{N-2} \varepsilon w_{N-2}}{\varepsilon} \\
& =(N-1)\left(w_{N-1}-w_{N-2}\right) \\
& <0
\end{aligned}
$$

where the last inequality comes from the fact that $w_{i}$ is a decreasing function in terms of $i$.

In all, $f^{\prime}(x)<0, \forall x \in[0,1]$. This completes the proof of the lemma.

Theorem 2: The condition for $x^{\star}=0$ to be an ESS is $\operatorname{Pr}\left(y_{1} \geq r\right) G-C \leq 0$.

Proof: According to (11-13), the utility that a peer using mixed strategy $x$ and the other peers use mixed strategy $x^{\star}=0$ can be written as

$$
\bar{U}(x, 0)=\bar{U}_{N}(0)+\left(\bar{U}_{A}(0)-\bar{U}_{N}(0)\right) x
$$

where $\bar{U}_{A}(0)=\operatorname{Pr}\left(y_{1} \geq r\right) G-C$ and $\bar{U}_{N}(0)=0$.

1) If $\operatorname{Pr}\left(y_{1} \geq r\right) G-C>0$, i.e. $\bar{U}_{A}(0)>\bar{U}_{N}(0)$, every peer will deviate to $x=1$ to obtain $\bar{U}_{A}(0)$ rather than $\bar{U}_{N}(0)$.

2) If $\operatorname{Pr}\left(y_{1} \geq r\right) G-C<0$, i.e. $\bar{U}_{A}(0)<\bar{U}_{N}(0)$, every peer will stay at $x=0$ to obtain $\bar{U}_{N}(0)$ rather than $\bar{U}_{A}(0)$.

3) If $\operatorname{Pr}\left(y_{1} \geq r\right) G-C=0$, i.e. $\bar{U}_{A}(0)=\bar{U}_{N}(0)$, then $\bar{U}(x, 0)=0 \forall x$, and $f(0)=\bar{U}_{A}(0)-\bar{U}_{N}(0)=0$. According to Lemma 1, we know that $f^{\prime}(x)<0$ $\forall x \in[0,1]$, so $f(x)=\bar{U}_{A}(x)-\bar{U}_{N}(x)<0$. In such a case, $\bar{U}(0, x)=\bar{U}_{N}(x)>\bar{U}(x, x)=\bar{U}_{N}(x)+\left(\bar{U}_{A}(x)-\right.$ $\left.\bar{U}_{N}(x)\right) x$, which means $x^{\star}=0$ is an ESS according to Definition 1 .

Therefore, $x^{\star}=0$ is an ESS only when $\operatorname{Pr}\left(y_{1} \geq r\right) G-C \leq 0$.

Theorem 3: The condition for $x^{\star}=1$ to be an ESS is $\operatorname{Pr}\left(y_{N} \geq r\right) G-\operatorname{Pr}\left(y_{N-1} \geq r\right) G \geq C$.

Proof: According to (11)-(13), the utility that a peer using mixed strategy $x$ and the other peers use mixed strategy $x^{\star}=1$ can be written as

$$
\bar{U}(x, 1)=\bar{U}_{N}(1)+\left(\bar{U}_{A}(1)-\bar{U}_{N}(1)\right) x
$$

where $\bar{U}_{A}(1)=\operatorname{Pr}\left(y_{N} \geq r\right) G-C$ and $\bar{U}_{N}(1)=\operatorname{Pr}\left(y_{N-1} \geq r\right) G$.

1) If $\operatorname{Pr}\left(y_{N} \geq r\right) G-\operatorname{Pr}\left(y_{N-1} \geq r\right) G<C$, i.e., $\bar{U}_{N}(1)>$ $\bar{U}_{A}(1)$, every peer will deviate to $x=0$ to obtain $\bar{U}_{N}(1)$ rather than $\bar{U}_{A}(1)$.

2) If $\operatorname{Pr}\left(y_{N} \geq r\right) G-\operatorname{Pr}\left(y_{N-1} \geq r\right) G>C$, i.e., $\bar{U}_{N}(1)<$ $\bar{U}_{A}(1)$, every peer will stay at $x=1$ to obtain $\bar{U}_{A}(1)$ rather than $\bar{U}_{N}(1)$.

3) If $\operatorname{Pr}\left(y_{N} \geq r\right) G-\operatorname{Pr}\left(y_{N-1} \geq r\right) G=C$, i.e. $\bar{U}_{N}(1)=$ $\bar{U}_{A}(1)$, then $\bar{U}(x, 1)=\bar{U}_{N}(1) \forall x$, and $f(1)=\bar{U}_{A}(1)-$ $\bar{U}_{N}(1)=0$. According to Lemma 1, we know that $f^{\prime}(x)<0 \forall x \in[0,1]$, so $f(x)=\bar{U}_{A}(x)-\bar{U}_{N}(x)>0$. 
In such a case, $\bar{U}(1, x)=\bar{U}_{N}(x)+\left(\bar{U}_{A}(x)-\bar{U}_{N}(x)\right) 1>$ $\bar{U}(x, x)=\bar{U}_{N}(x)+\left(\bar{U}_{A}(x)-\bar{U}_{N}(x)\right) x$ which means $x^{\star}=1$ is an ESS according to Definition 1.

Therefore, $x^{\star}=1$ is an ESS only when $\operatorname{Pr}\left(y_{N} \geq r\right) G-$ $\operatorname{Pr}\left(y_{N-1} \geq r\right) G \geq C$.

Theorem 4: If $x^{\star} \in(0,1)$ is a solution to $\bar{U}_{A}(x)=\bar{U}_{N}(x)$, then $x^{\star}$ is an ESS.

Proof: Let $\bar{U}_{i}\left(x, x^{\star}\right)$ be the utility of player $i$ when player $i$ uses mixed strategy $x$ and other users use mixed strategy $x^{\star}$. Then, we have

$$
\bar{U}_{i}\left(x, x^{\star}\right)=x \bar{U}_{A}\left(x^{\star}\right)+(1-x) \bar{U}_{N}\left(x^{\star}\right) .
$$

Since $x^{\star}$ is a solution to $\bar{U}_{A}(x)=\bar{U}_{N}(x)$, we have $\bar{U}_{A}\left(x^{\star}\right)=$ $\bar{U}_{N}\left(x^{\star}\right)$. Therefore, (20) becomes

$$
\bar{U}_{i}\left(x, x^{\star}\right)=\bar{U}_{A}\left(x^{\star}\right)=\bar{U}_{i}\left(x^{\star}, x^{\star}\right),
$$

which means $x^{\star}$ satisfies the equilibrium condition shown in Definition 1.

Moreover, according to (13), we have

$$
\bar{U}_{i}(x, x)=\bar{U}_{N}(x)+\left(\bar{U}_{A}(x)-\bar{U}_{N}(x)\right) x
$$

and

$$
\bar{U}_{i}\left(x^{\star}, x\right)=\bar{U}_{N}(x)+\left(\bar{U}_{A}(x)-\bar{U}_{N}(x)\right) x^{\star} .
$$

Therefore, we have

$$
\bar{U}_{i}\left(x^{\star}, x\right)-\bar{U}_{i}(x, x)=\left(\bar{U}_{A}(x)-\bar{U}_{N}(x)\right)\left(x^{\star}-x\right) .
$$

From Lemma 1, we know that $f(x)=\bar{U}_{A}(x)-\bar{U}_{N}(x)$ is a monotonically decreasing function. Since $\bar{U}_{A}\left(x^{\star}\right)=\bar{U}_{N}\left(x^{\star}\right)$, $\bar{U}_{A}(x)-\bar{U}_{N}(x)>0$ if $x<x^{\star}$, and $\bar{U}_{A}(x)-\bar{U}_{N}(x)<0$ if $x>x^{\star}$. Therefore, $\left(\bar{U}_{A}(x)-\bar{U}_{N}(x)\right)\left(x^{\star}-x\right)>0, \forall x \neq x^{\star}$, that is

$$
\bar{U}_{i}\left(x^{\star}, x\right)>\bar{U}_{i}(x, x), \forall x \neq x^{\star}
$$

which means $x^{\star}$ satisfies the stability condition shown in Definition 1.

According to (21) and (25), we know that $x^{\star}$ is an ESS.

\section{Agents Selection Within A Heterogeneous GROUP}

In this section, we will discuss how to select agents within a heterogeneous group where the costs of the peers acting as agents are different.

Let $x_{i, a_{i}}$ stand for the probability of peer $u_{i}$ using pure strategy $a_{i} \in \mathcal{A}$. By replicator dynamics, the evolution dynamics of $x_{i, a_{i}}$ are given by the following differential equation:

$$
\dot{x}_{i, a_{i}}=\eta\left[\bar{U}_{i}\left(a_{i}, x_{-i}\right)-\bar{U}_{i}\left(x_{i}\right)\right] x_{i, a_{i}}
$$

where $\bar{U}_{i}\left(a_{i}, x_{-i}\right)$ is the average payoff of peer $u_{i}$ using pure strategy $a_{i}, \bar{U}_{i}\left(x_{i}\right)$ is the average payoff of peer $u_{i}$ using mixed strategy $x_{i}$, and $\eta$ is a positive scale factor.

Since it is generally very difficult to represent $\bar{U}_{i}\left(a_{i}, x_{-i}\right)$ and $\bar{U}_{i}\left(x_{i}\right)$ in a compact form, in the following, we first analyze a two-player game to gain some insight. Then, we generalize the observation in the two-player game to the multi-player game.
TABLE I

UTILITy TABle OF A TWO-Player GAME

\begin{tabular}{|c|c|c|}
\hline & "A" & " $N "$ \\
\hline " $A$ " & $\left(B_{2}-C_{1}, B_{2}-C_{2}\right)$ & $\left(B_{1}-C_{1}, B_{1}\right)$ \\
\hline$" N "$ & $\left(B_{1}, B_{1}-C_{2}\right)$ & $(0,0)$ \\
\hline
\end{tabular}

\section{A. Two-Player Game}

Let $x_{1}$ and $x_{2}$ be the probability of $u_{1}$ and $u_{2}$ being an agent, respectively. Let $B_{1}=\operatorname{Pr}\left(y_{1} \geq r\right) G$ and $B_{2}=\operatorname{Pr}\left(y_{2} \geq\right.$ $r) G$. Then, the payoff matrix of $u_{1}$ and $u_{2}$ can be written as in Table I. Therefore, the average payoff $\bar{U}_{1}\left(A, x_{2}\right)$ can be computed by

$$
\bar{U}_{1}\left(A, x_{2}\right)=\left(B_{2}-C_{1}\right) x_{2}+\left(B_{1}-C_{1}\right)\left(1-x_{2}\right)
$$

and the average payoff $\bar{U}_{1}\left(x_{1}\right)$ becomes

$$
\begin{aligned}
\bar{U}_{1}\left(x_{1}\right) & =\left(B_{2}-C_{1}\right) x_{1} x_{2} \\
& +\left(B_{1}-C_{1}\right) x_{1}\left(1-x_{2}\right)+B_{1}\left(1-x_{1}\right) x_{2} .
\end{aligned}
$$

According to (26), the replicator dynamics equation of $u_{1}$ is given by

$$
\dot{x}_{1}=\eta x_{1}\left(1-x_{1}\right)\left[B_{1}-C_{1}-\left(2 B_{1}-B_{2}\right) x_{2}\right] .
$$

Similarly, the replicator dynamics equation of $u_{2}$ can be computed by

$$
\dot{x}_{2}=\eta x_{2}\left(1-x_{2}\right)\left[B_{1}-C_{2}-\left(2 B_{1}-B_{2}\right) x_{1}\right] .
$$

At equilibrium, we know that $\dot{x}_{1}=0$ and $\dot{x}_{2}=0$. According to (29) and (30), we can get five equilibria: $(0,0),(0,1),(1,0)$, $(1,1)$, and the mixed strategy equilibrium $\left(\frac{B_{1}-C_{2}}{2 B_{1}-B_{2}}, \frac{B_{1}-C_{1}}{2 B_{1}-B_{2}}\right)$.

According to [32], if an equilibrium of the replicator dynamics equations is a locally asymptotically stable point in a dynamic system, it is an ESS. Therefore, by viewing (29) and (30) as a nonlinear dynamic system and analyzing the corresponding Jacobian matrix, we can examine whether the five equilibria are ESSs. By taking partial derivatives of (29) and (30), the Jacobian matrix can be written as

$$
J=\left[\begin{array}{ll}
\frac{\partial \dot{x}_{1}}{\partial x_{1}} & \frac{\partial \dot{x}_{1}}{\partial x_{2}} \\
\frac{\partial \dot{x}_{2}}{\partial x_{1}} & \frac{\partial \dot{x}_{2}}{\partial x_{2}}
\end{array}\right]=\eta\left[\begin{array}{ll}
J_{11} & J_{12} \\
J_{21} & J_{22}
\end{array}\right]
$$

where $J_{11}=\left(1-2 x_{1}\right)\left(B_{1}-C_{1}-\left(2 B_{1}-B_{2}\right) x_{2}\right), J_{12}=-x_{1}(1-$ $\left.x_{1}\right)\left(2 B_{1}-B_{2}\right), J_{21}=-x_{2}\left(1-x_{2}\right)\left(2 B_{1}-B_{2}\right)$, and $J_{22}=(1-$ $\left.2 x_{2}\right)\left(B_{1}-C_{2}-\left(2 B_{1}-B_{2}\right) x_{1}\right)$.

The asymptotical stability requires that $\operatorname{det}(J)>0$ and $\operatorname{tr}(J)<0$ [32]. Substituting the five equilibria, i.e. $(0,0)$, $(0,1),(1,0),(1,1)$, and $\left(\frac{B_{1}-C_{2}}{2 B_{1}-B_{2}}, \frac{B_{1}-C_{1}}{2 B_{1}-B_{2}}\right)$, to (31), we conclude that the following.

1) If $B_{2}-B_{1}-C_{1}>0$ and $B_{2}-B_{1}-C_{2}>0$, there is a unique ESS $(1,1)$, where both $u_{1}$ and $u_{2}$ converge to be agents.

2) Elseif $B_{2}-B_{1}-C_{1}>0$ and $B_{2}-B_{1}-C_{2}<0$, there is a unique $\operatorname{ESS}(1,0)$, where $u_{1}$ converges to be an agent and $u_{2}$ converges to be a free-rider.

3) Elseif $B_{2}-B_{1}-C_{1}<0$ and $B_{2}-B_{1}-C_{2}>0$, there is a unique $\operatorname{ESS}(0,1)$, where $u_{2}$ converges to be an agent and $u_{1}$ converges to be a free-rider. 


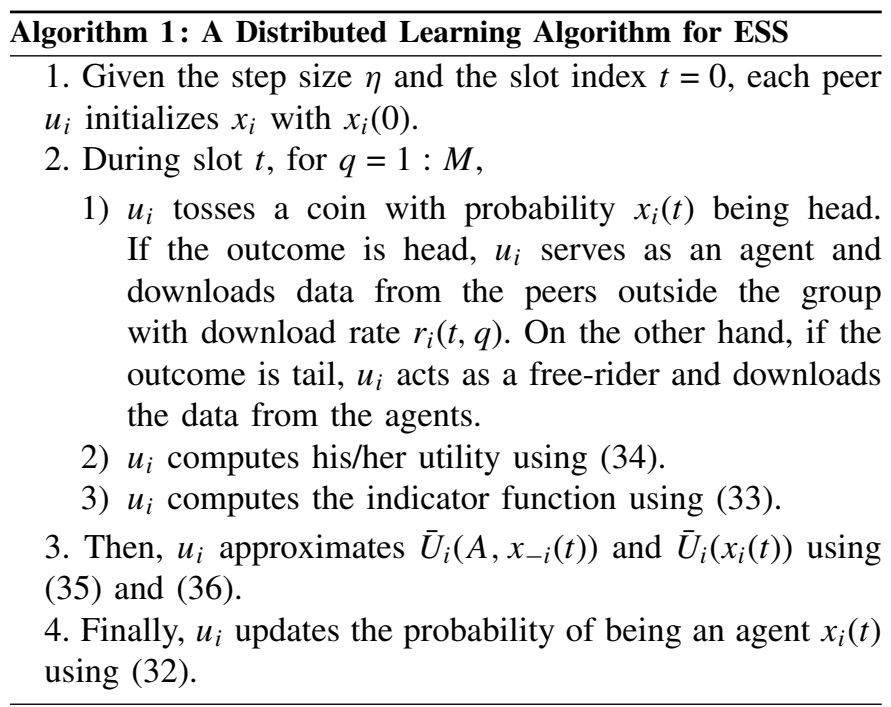

4) Else, there are two ESSs $(0,1)$ and $(1,0)$, where the converged strategy profiles depends on the initial strategy profiles.

From the above analysis, we can see that when the gain of being an agent $\left(B_{2}-B_{1}\right)$ is greater than the cost of being an agent $\left(C_{1}\right.$ or $\left.C_{2}\right)$, the peer tends to be an agent. And the peer with a higher cost tends to be a free-rider and rely on the peer with a lower cost.

\section{B. Multi-Player Game}

From the analysis of the two-player game, we can infer that the peer with a higher cost $\left(C_{i}\right)$ tends to take advantage of the peer with a lower cost. This observation can be extended to multi-player game. If there are more than two peers in the game, the strategy of the peers with higher $C_{i} \mathrm{~s}$ will converge to " $N$ " with a greater probability. The peers with lower $C_{i} \mathrm{~s}$ tend to be agents since they suffer relatively heavier losses if no one serves as an agent.

\section{A Distributed LEARning AlgORITHM FOR ESS}

From the previous two sections, we can see that the ESS can be found by solving the replicator dynamics equations [(14) or (26)]. However, solving the replicator dynamics equations require the exchange of private information and strategies adopted by other peers. In this section, we will present a distributed learning algorithm that can gradually converge to ESS without information exchange.

We first discretize the replicator dynamics equation shown in (26) as

$$
x_{i}(t+1)=x_{i}(t)+\eta\left[\bar{U}_{i}\left(A, x_{-i}(t)\right)-\bar{U}_{i}\left(x_{i}(t)\right)\right] x_{i}(t)
$$

where $t$ is the slot index and $x_{i}(t)$ is the probability of $u_{i}$ being an agent during slot $t$. Here, we assume that each slot can be further divided into $M$ subslots and each peer can choose to be an agent or not at the beginning of each subslot.

From (32), we can see that in order to update $x_{i}(t+1)$, we need to first compute $\bar{U}_{i}\left(A, x_{-i}(t)\right)$ and $\bar{U}_{i}\left(x_{i}(t)\right)$. Let us define an indicator function $\mathbf{1}_{i}(t, k)$ as

$$
\mathbf{1}_{i}(t, q)= \begin{cases}1 & \text { if } u_{i} \text { is an agent at subslot } q \text { in slot } t \\ 0 & \text { else }\end{cases}
$$

where $q$ is the subslot index.

The immediate utility of $u_{i}$ at subslot $q$ in slot $t$ can be computed by

$$
U_{i}(t, q)= \begin{cases}G-C_{i} & \text { if } u_{i} \text { is an agent and } r^{t} \geq r \\ -C_{i} & \text { if } u_{i} \text { is an agent and } r^{t}<r \\ G & \text { if } u_{i} \text { is not an agent and } r^{t} \geq r \\ 0 & \text { if } u_{i} \text { is not an agent and } r^{t}<r\end{cases}
$$

where $r^{t}$ is the total download rate of the agents and $r$ is the source rate.

Then, $\bar{U}_{i}\left(A, x_{-i}(t)\right)$ can be approximated using

$$
\bar{U}_{i}\left(A, x_{-i}(t)\right)=\frac{\sum_{q=1}^{M} U_{i}(t, q) \mathbf{1}_{i}(t, q)}{\sum_{q=1}^{M} \mathbf{1}_{i}(t, q)} .
$$

Similarly $\bar{U}_{i}\left(x_{i}(t)\right)$ can be approximated as

$$
\bar{U}_{i}\left(x_{i}(t)\right)=\frac{1}{M} \sum_{q=1}^{M} U_{i}(t, q)
$$

Based on (32)-(36), $u_{i}$ can gradually learn the ESS. In Algorithm 1, we summarize the detailed procedures of the proposed distributed learning algorithm.

\section{Simulation Results}

In all simulations, the parameters $G, r^{L}$, and $r^{U}$ are set to be 1,50 , and 800 , respectively. For convenience, in the rest of this paper, we denote the centralized approach maximizing the social welfare shown in (7) as MSW-C, the distributed approach maximizing the social welfare shown in (9) as MSW-D, and the ESS-based approach as ESS-D. We compare the proposed methods with the traditional P2P non-cooperation method, denoted as Non-Coop. In Non-Coop, each peer acts as an individual and randomly selects some peers for downloading video streams. Such a protocol has been widely used in the existing P2P systems, e.g., Coolstreaming [7] and PPLive [8].

In the first simulation, we show the social welfare (the sum of all peers' utilities) comparison among different approaches, where we assume that there are 20 homogenous peers and the cost $C$ is 0.1 . As show in Fig. 3, MSW-C achieves the best social welfare performance since its objective function is to maximize the social welfare with pure strategy. By using the mixed strategy to maximize the social welfare, MSW-D achieves the second best social welfare performance. However, as discussed in Section III. B, the solution to MSW-D is not stable. With ESS-D, a stable NE solution can be obtained at the cost of a slight loss in social welfare. Nevertheless, all three proposed algorithms perform much better than the NonCoop method. In Non-Coop, the social welfare performance decreases linearly in terms of the source rate. With cooperation and adaptively selecting the proper number of agents, all three proposed algorithms can preserve a high social welfare performance even with a large source rate. 


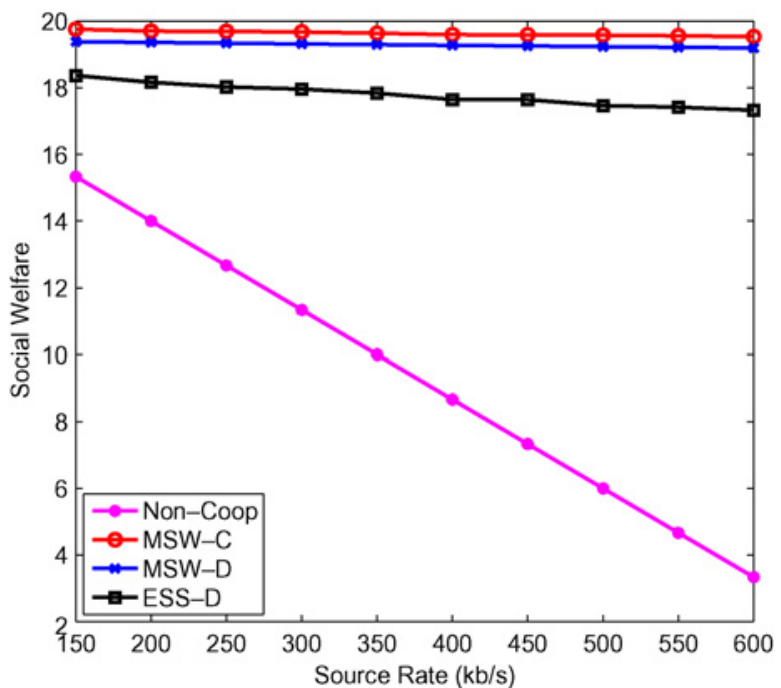

Fig. 3. Social welfare comparison among Non-Coop, MSW-C, MSW-D, and ESS-D.

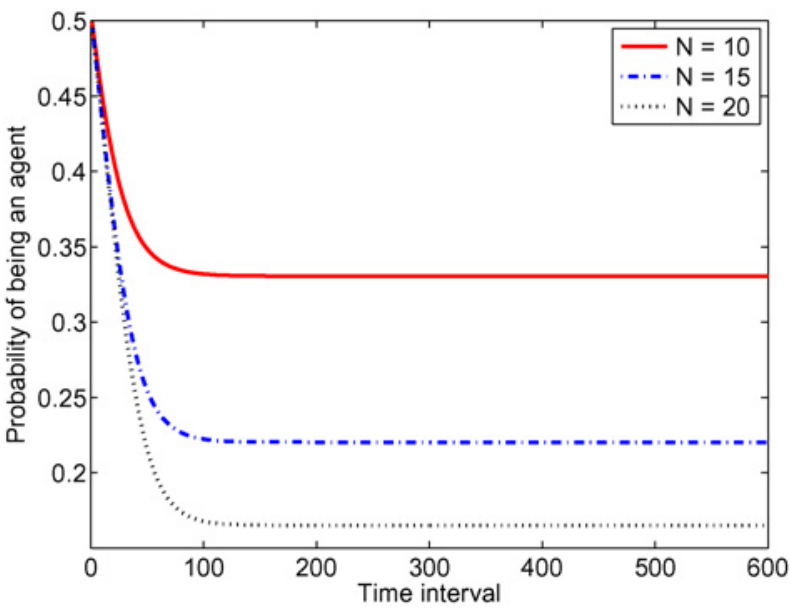

Fig. 4. Behavior dynamic of a homogeneous group of peers.

In the second simulation, we evaluate the convergence property of the ESS-D. In Fig. 4, we show the replicator dynamic of the cooperation streaming game with homogeneous peers, where $C=0.1$ and $r=500$. We can see that starting from a high initial value, all peers gradually reduce their probabilities of being an agent since being a free-rider more often can bring a higher payoff. However, since too low a probability of being an agent increases the chance of having no peer be an agent, the probability of being an agent will finally converge to a certain value which is determined by the number of peers.

In Fig. 5, we show the replicator dynamic of the cooperation streaming game with 20 heterogeneous peers, where $r=500$ and the $\operatorname{cost} C_{i}$ is randomly chosen from $[0.1,0.3]$. We further assume that $C_{i}$ is monotonically increasing in $i$ where $u_{1}$ has the lowest cost and $u_{20}$ has the highest cost. From Fig. 5, we can see that the peers with lower costs $\left(u_{1}, u_{2}\right.$, and $u_{3}$ in this simulation) converge to be an agent while the peers with higher costs $\left(u_{4}-u_{20}\right.$ in this simulation) converge to be a free-rider. This observation coincides with our conclusion in Section IV-B, which is the following: the peers with lower
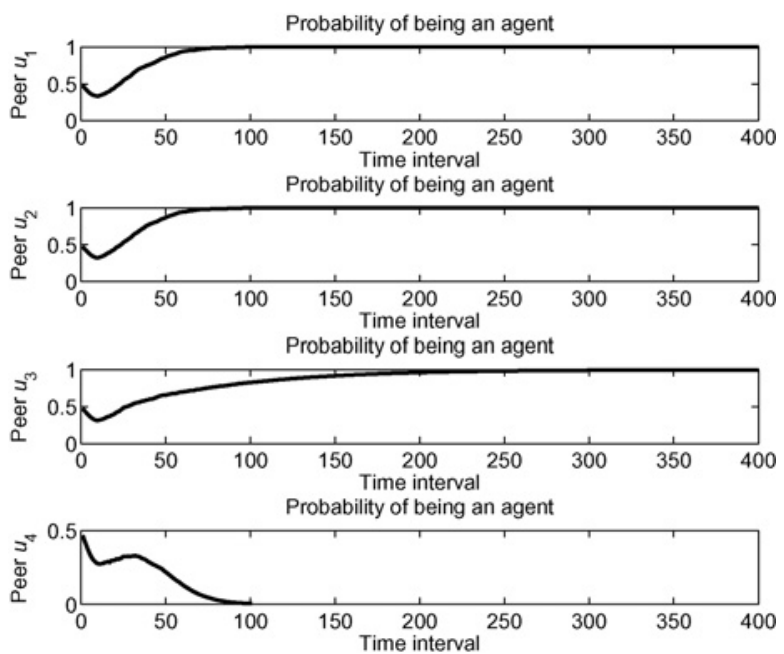

Fig. 5. Behavior dynamic of a heterogeneous group of peers.

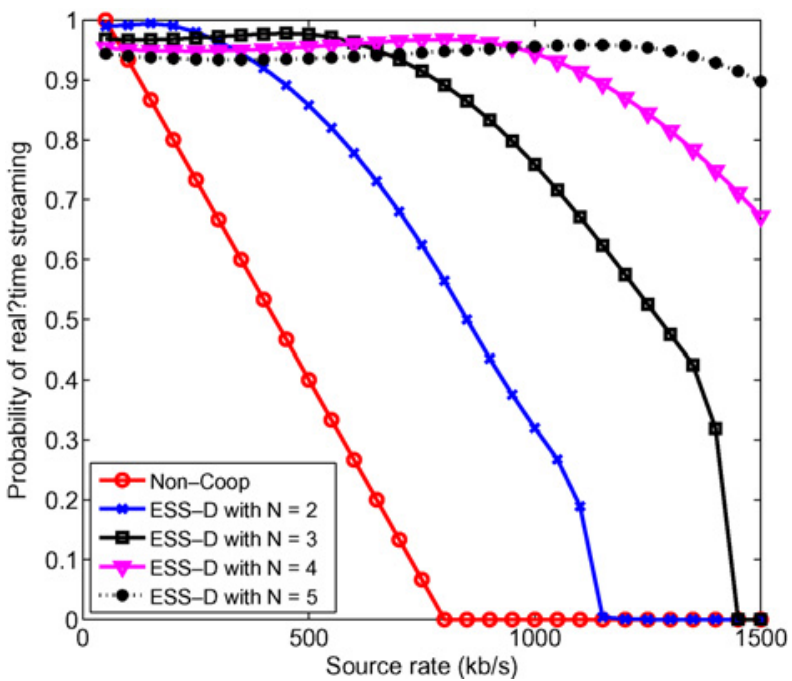

Fig. 6. Probability of real-time streaming comparison between Non-Coop and ESS-D.

costs tend to be an agent since they suffer relatively higher losses if no one serves as an agent. Note that due to the space limitation, we only show the behavior dynamics of $u_{1}-u_{4}$. All other peers $u_{5}-u_{20}$ have the similar behavior dynamics with $u_{4}$, and they all converge to be free-riders.

In the third simulation, we compare the performance of Non-Coop and ESS-D in terms of the probability of real-time streaming, which is defined as the probability that the total download rate is greater than the source rate. The simulation results are shown in Fig. 6. We can see that with cooperation, the probability of real-time streaming can be significantly improved especially at the high source rate region. We also find that at the high source rate region, the probability of realtime streaming increases as $N$ increases.

The visual quality comparison between Non-Coop and ESS-D is shown in Fig. 7. In this simulation, we fix the probability of real-time streaming to be 0.85 . According to Fig. 6, we can see that the corresponding source rates for "Non-Coop," "ESS-D with N=2," "ESS-D with $\mathrm{N}=3$," and 


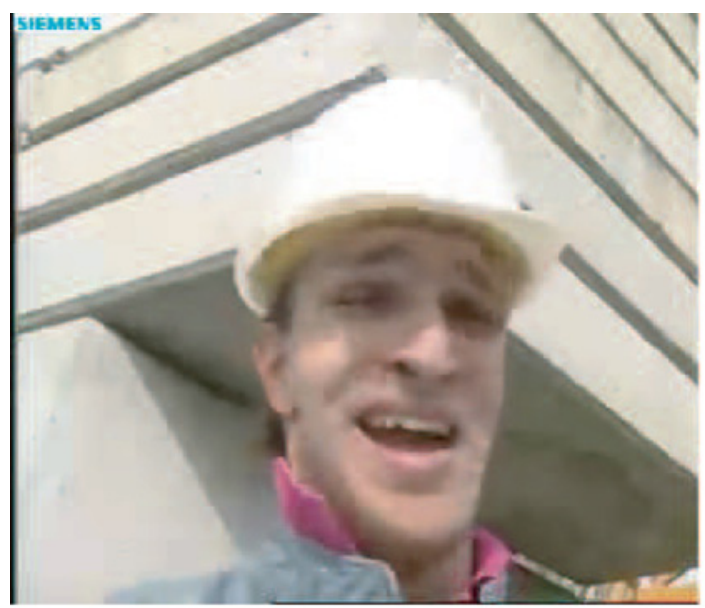

(a)

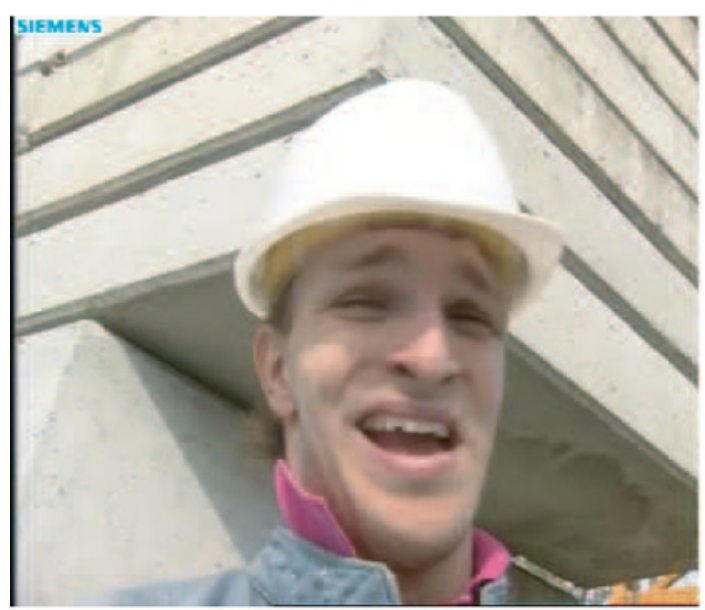

(c)

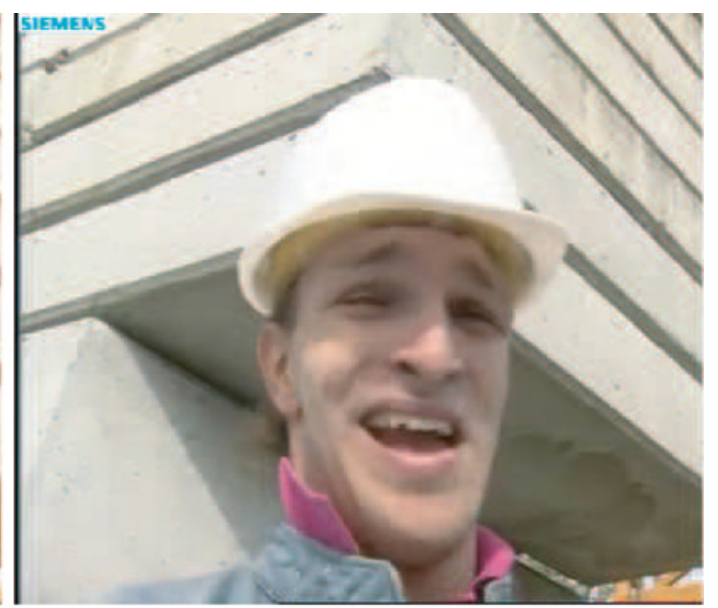

(b)

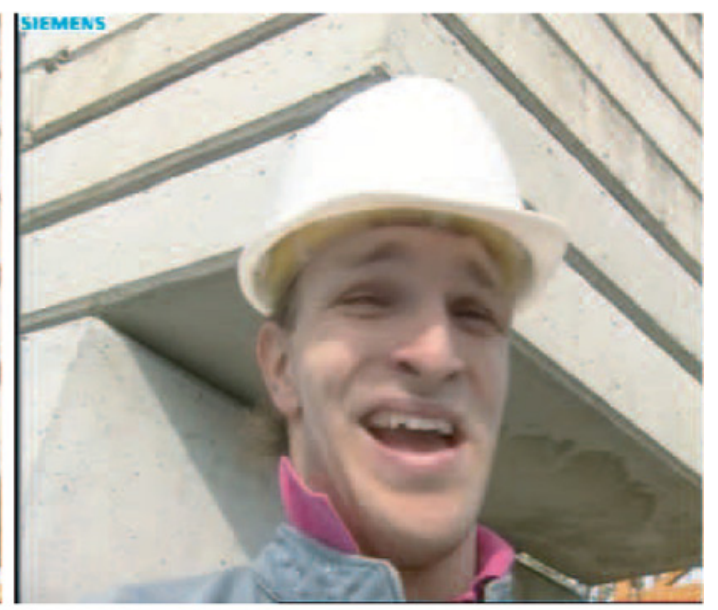

(d)

Fig. 7. Visual quality comparison. (a) Non-Coop. (b) ESS-D with $N=2$. (c) ESS-D with $N=3$. (d) ESS-D with $N=4$.

"ESS-D with N=4" are around $100 \mathrm{~kb} / \mathrm{s}, 300 \mathrm{~kb} / \mathrm{s}, 520 \mathrm{~kb} / \mathrm{s}$, and $720 \mathrm{~kb} / \mathrm{s}$, respectively. By setting the above source rates as the target bitrates, we encode the Foreman sequence with CIF format using H.264 encoder. From Fig. 7, we can see that the video visual quality with the proposed ESS-D is much better than that with Non-Coop.

Then, we show the simulation result of the source rate vs. the utility. As shown in Fig. 8, without cooperation, if the peer requires a utility around 0.8 , the source rate cannot be larger than $130 \mathrm{~kb} / \mathrm{s}$. However, with cooperation, the source rate can be more than $400 \mathrm{~kb} / \mathrm{s}$ even when there are only 2 peers. Therefore, with cooperation, the peers can enjoy much higher quality video with the same utility.

In the fourth simulation, we consider the case that the peers in the same group are viewing multiple channels with $L$ being the number of the channels. We assume that the source rate is the same for all channels and there are 20 homogenous peers with the cost $C=0.1$. Similar to the viewupload decoupling (VUD) scheme [33], the uploading and downloading are decoupled in the proposed ESS-D algorithm in this case. We allow cooperation among all the peers where the agent may download source data that he/she is not viewing. As shown in Fig. 9, without cooperation, if the peer requires a utility around 0.8 , the source rate cannot be larger than 130

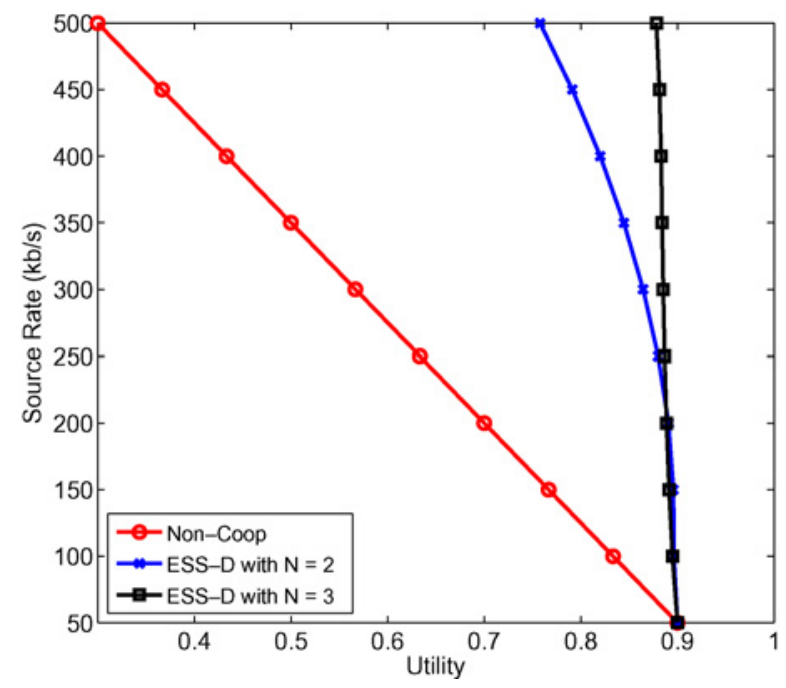

Fig. 8. Single-source rate comparison between Non-Coop and ESS-D.

$\mathrm{kb} / \mathrm{s}$ in the Non-Coop method. However, with the proposed ESS-D algorithm, the source rate can be around $240 \mathrm{~kb} / \mathrm{s}$ even when the peers are view 8 different channels. This phenomenon fully demonstrates the efficiency of the proposed method. 


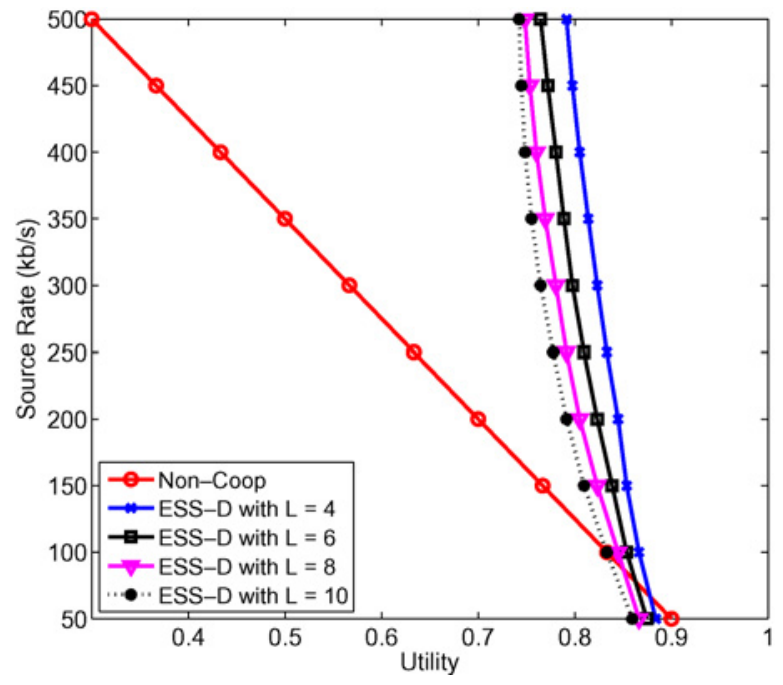

Fig. 9. Multi-source rate comparison between Non-Coop and ESS-D.

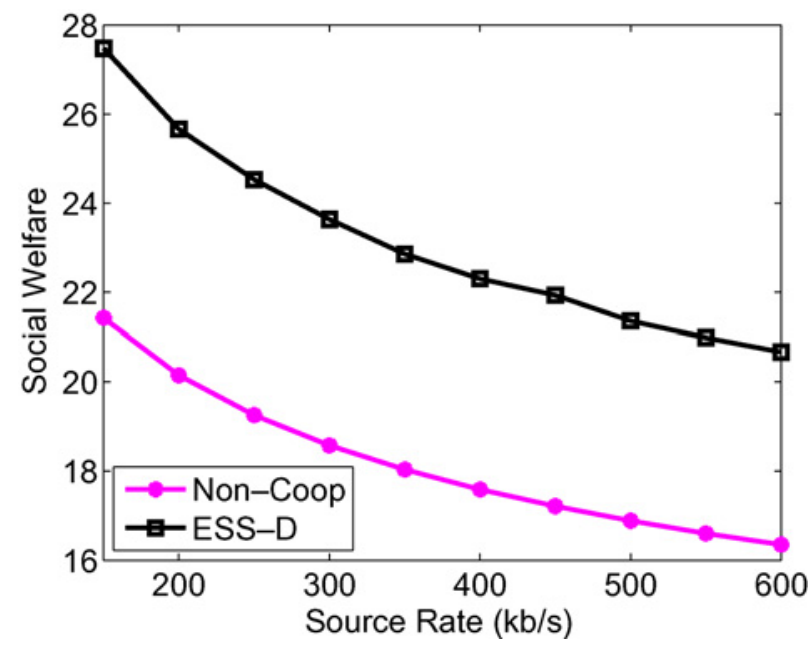

Fig. 10. Social welfare comparison between Non-Coop and ESS-D when the utility function is defined as (37).

In the last simulation, we consider the scenario when there is buffering effect. In such a scenario, the gain in the utility will not drop to zero when the total download rate is smaller than the source rate. Instead, the gain should maintain a positive value due to the existence of buffers. One possible utility function that considers the buffering effect is

$$
\begin{aligned}
& U_{A, i}(k)=\frac{1}{\ln (r)} E\left[\ln \left(y_{k}\right)\right] G-C_{i} \forall k \in[1, N] \\
& U_{N, i}(k)= \begin{cases}\frac{1}{\ln (r)} E\left[\ln \left(y_{k}\right)\right] G & \text { if } k \in[1, N-1] \\
0 & \text { if } k=0 .\end{cases}
\end{aligned}
$$

From the above utility function, we can see that for any given source rate $r$, the gain increases as the total download rate $y_{k}$ increases. Moreover, since the probability of playback delay becomes smaller with more data in the buffer, a certain increase in the high $y_{k}$ region should lead to a less significant gain than that in the low $y_{k}$ region [34]. Here, we use the $\ln ($. function to characterize such properties. Nevertheless, other functions that have similar properties can also be used.
The social welfare comparison between Non-Coop and ESS-D with the utility function in (37) is shown in Fig. 10. From Fig. 10, we can see that when the utility function in (37) is used, the social welfare performance of Non-Coop no longer decreases linearly in terms of the source rate. This phenomenon is mainly because, with the existence of buffers, the gain will not drop to zero when the total download rate is smaller than the source rate. Nevertheless, ESS-D can still lead to a much higher social welfare performance for all source rates, compared with Non-Coop. Moreover, we should notice that all the analysis in Section III is still applicable to the utility function in (37).

\section{CONCLUSION}

In this paper, we proposed a cooperative streaming scheme to address the network inefficiency problem encountered by the traditional non-cooperative P2P schemes. We answer the question of how a group of selfish peers with large intragroup upload and download bandwidths cooperate with each other to achieve better streaming performance by formulating the problem as an evolutionary game and deriving the ESS for every peer. We further proposed a distributed learning algorithm for each peer to converge to the ESS by learning from his/her own past payoff history. From the simulation results, we can see that compared with the traditional noncooperative $\mathrm{P} 2 \mathrm{P}$ schemes, the proposed algorithm achieves much better social welfare, higher probability of real-time streaming, and better video quality (higher source rate). Moreover, by incorporating with the recent proposed VUD scheme, the proposed cooperative streaming scheme allows the peers who are viewing different videos to cooperate with each other and mutually improve the streaming performance.

\section{APPENDIX}

\section{A. Proof of Theorem 1}

Proof: Let $\hat{r}_{l}=\frac{r_{l}-r^{L}}{r^{U}-r^{L}}, \forall l$, then $\hat{r}_{1}, \hat{r}_{2}, \ldots, \hat{r}_{k}$ are i.i.d. uniformly distributed with $[0,1]$. And the characteristic function of $\hat{r}_{l}$ is given by

$$
\phi(t)=\frac{i\left(1-e^{i t}\right)}{t} .
$$

Let $\hat{y}_{k}=\sum_{l=1}^{k} \hat{r}_{l}$, then the characteristic function of $\hat{y}_{k}$ can be computed by

$$
\phi_{\hat{y}_{k}}(t)=\left(\frac{i\left(1-e^{i t}\right)}{t}\right)^{k} .
$$

Therefore, the density function of $\hat{y}_{k}$ is

$$
\begin{aligned}
f_{\hat{y}_{k}}(y) & =\mathcal{F}_{t}^{-1}\left[\left(\frac{i\left(1-e^{i t}\right)}{t}\right)^{k}\right](y) \\
& =\frac{1}{2(k-1) !} \sum_{l=0}^{k}(-1)^{l}\left(\begin{array}{l}
k \\
l
\end{array}\right) \operatorname{sgn}(y-l)(y-l)^{k-1} .
\end{aligned}
$$


Since $\operatorname{Pr}\left(y_{k} \geq r\right)=\operatorname{Pr}\left(\hat{y}_{k} \geq \hat{r}\right)$, according to (40), we have

$$
\begin{aligned}
& \operatorname{Pr}\left(y_{k} \geq r\right) \\
& \quad=\operatorname{Pr}\left(\hat{y}_{k} \geq \hat{r}\right)=\int_{\hat{r}}^{\infty} f_{\hat{y}_{k}}(y) d y \\
& \quad=\frac{1}{2 k !} \sum_{l=0}^{k}(-1)^{l}\left(\begin{array}{l}
k \\
l
\end{array}\right)\left[(k-l)^{k}-\operatorname{sgn}(\hat{r}-l)(\hat{r}-l)^{k}\right] .
\end{aligned}
$$

When $k$ is sufficiently large, according to the central limit theory, the distribution of $\hat{y}_{k}$ can be approximated as Gaussian distribution $N\left(\frac{k}{2}, \frac{k}{12}\right)$. Therefore, we have

$$
\operatorname{Pr}\left(y_{k} \geq r\right)=\operatorname{Pr}\left(\hat{y}_{k} \geq \hat{r}\right) \approx Q\left(\frac{\hat{r}-\frac{k}{2}}{\sqrt{\frac{k}{12}}}\right) .
$$

\section{REFERENCES}

[1] Accustream iMedia Research Homepage [Online]. Available: http://www.accustreamresearch.com

[2] YouTube [Online]. Available: http://www.youtube.com

[3] S. Deering and D. Cheriton, "Multicast routing in datagram internetworks and extended lans," ACM Trans. Comput. Syst., vol. 8, no. 2, pp. 85-111, 1990.

[4] L. Kontothanassis, R. Sitaraman, J. Wein, D. Hong, R. Kleinberg, B. Mancuso, D. Shaw, and D. Stodolsky, "A transport layer for live streaming in a content delivery network," IEEE Proc., vol. 92, no. 9, pp. 1408-1419, Sep. 2004.

[5] Y. Liu, Y. Guo, and C. Liang, "A survey on peer-to-peer video streaming systems," J. Peer-to-Peer Netw. Applicat., vol. 1, no. 1, pp. 18-28, 2008

[6] Y. Chu, S. G. Rao, and H. Zhang, "A case for end system multicast," in Proc. ACM SIGMETRICS, 2000, pp. 1-12.

[7] X. Zhang, J. Liu, B. Li, and T.-S. P. Yum, "Coolstreaming: A data-driven overlay network for peer-to-peer live media streaming," in Proc. IEEE Conf. Comput. Commun. (INFOCOM), 2005, pp. 2102-2111.

[8] PPLive [Online]. Available: http://www.pplive.com

[9] PPStream [Online]. Available: http://www.ppstream.com

[10] UUSee [Online]. Available: http://www.uusee.com

[11] Sopcast [Online]. Available: http://www.sopcast.com

[12] X. Hei, C. Liang, J. Liang, Y. Liu, and K. Ross, "A measurement study of a large-scale P2P IPTV system," IEEE Trans. Multimedia, vol. 9, no. 8, pp. 1672-1687, Dec. 2007.

[13] H. Xie, Y. R. Yang, A. Krishnamurthy, Y. Liu, and A. Siberschatz, "P4P: Provider portal for applications," in Proc. ACM SIGCOMM, 2008, pp. 351-362.

[14] T. Karagiannis, P. Rodriguez, and K. Papagiannaki, "Should internet service providers fear peer-assisted content distribution?" in Proc. Internet Meas. Conf., 2005, p. 6.

[15] S. Seetharaman and M. Ammar, "Characterizing and mitigating interdomain policy violations in overlay routes," in Proc. IEEE Int. Conf. Netw. Protocols, 2006, pp. 259-268.

[16] M. Xiao and D. Xiao, "Understanding peer behavior and designing incentive mechanism in peer-to-peer networks: An analytical model based on game theory," in Proc. ICA3PP, 2007, pp. 368-379.

[17] A. Habib and J. Chuang, "Service differentiated peer selection: An incentive mechanism for peer-to-peer media streaming," IEEE Trans. Multimedia, vol. 8, no. 3, pp. 610-621, Jun. 2006.

[18] H. V. Madhyastha, T. Isdal, M. Piatek, C. Dixon, T. Anderson, A. Krishnamurthy, and A. Venkataramani, "iplane: An information plane for distributed services," in Proc. 7th Symp. OSDI, 2006, pp. 367-380.

[19] D. Purandare and R. Guha, "An alliance based peering scheme for $\mathrm{p} 2 \mathrm{p}$ live media streaming," IEEE Trans. Multimedia, vol. 9, no. 8, pp. 16331644, Dec. 2007.

[20] V. Vishumurthy, S. Chandrakumar, and E. Sirer, "KARMA: A secure economic framework for peer-to-peer resource sharing," in Proc. Workshop Econ. Peer-to-Peer Syst., 2003.

[21] P. Golle, K. Leyton-Brown, and I. Mironov, "Incentive for sharing in peer-to-peer networks," in Proc. ACM Conf. Electron. Commerce, 2001, pp. $75-87$.
[22] S. Marti and H. Garcia-Molina, "Limited reputation sharing in P2P systems," in Proc. 5th ACM Conf. Electron. Commerce, 2004, pp. 91101 .

[23] M. Gupta, P. Judge, and M. Ammar, "A reputation system for peer-topeer networks," in Proc. ACM 13th Int. Workshop Netw. Operating Syst. Support Digital Audio Video, 2003, pp. 144-152.

[24] B. Wang, Y. Wu, and K. J. R. Liu, "Game theory for cognitive radio networks: An overview," Comput. Netw., vol. 54, no. 14, pp. 2537-2561, Oct. 2010.

[25] H. Zhao, W. Lin, and K. J. R. Liu, "Behavior modeling and forensics for multimedia social networks: A case study in multimedia fingerprinting," IEEE Signal Process. Mag., vol. 26, no. 1, pp. 118-139, Jan. 2009.

[26] I. Ahmad and J. Luo, "On using game theory for perceptually tuned rate control algorithm for video coding," IEEE Trans. Circuits Syst. Video Technol., vol. 16, no. 2, pp. 202-208, Feb. 2006.

[27] Y. Chen, B. Wang, and K. J. R. Liu, "Multi-user rate allocation games for multimedia communications," IEEE Trans. Commun., vol. 11, no. 6, pp. 1170-1181, Oct. 2009.

[28] W. S. Lin, H. V. Zhao, and K. J. R. Liu, "Incentive cooperation strategies for peer-to-peer live multimedia streaming social networks," IEEE Trans. Multimedia, vol. 11, no. 3, pp. 396-412, Apr. 2009.

[29] C. Huang, J. Li, and K. W. Ross, "Can internet video-on-demand be profitable?" in Proc. ACM SIGCOMM, 2007, pp. 133-144.

[30] J. M. Smith, Evolutionary and the Theory of Games. Cambridge, MA: Cambridge University Press, 1982.

[31] B. Wang, K. J. R. Liu, and T. C. Clancy, "Evolutionary cooperative spectrum sensing game: How to collaborate," IEEE Trans. Commun., vol. 58 , no. 3, pp. 890-900, Mar. 2010.

[32] R. Cressman, Evolutionary Dynamics and Extensive Form Games. Cambridge, MA: MIT Press, 2003.

[33] D. Wu, C. Liang, Y. Liu, and K. W. Ross, "View-upload decoupling: A redesign of multi-channel P2P video systems," in Proc. IEEE INFOCOM, 2009, pp. 2760-2730

[34] Y. Chen, Y. Wu, B. Wang, and K. Liu, "Spectrum auction games for multimedia streaming over cognitive radio networks," IEEE Trans. Commun., vol. 58, no. 8, pp. 2381-2390, Aug. 2010.

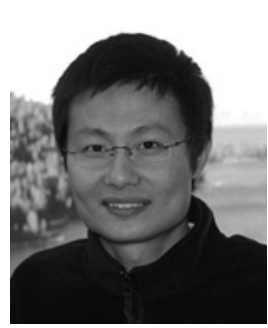

Yan Chen (S'06) received the Bachelors degree from the University of Science and Technology of China, Hefei, Anhui, China, in 2004, and the M.Phil. degree from the Hong Kong University of Science and Technology, Clear Water Bay, Hong Kong, in 2007. He is currently pursuing the Ph.D. degree from the Department of Electrical and Computer Engineering, University of Maryland, College Park.

His current research interests include game theoretical modeling for multimedia social networks, multimedia signal processing, cooperative multimedia communication and networking, and multimedia forensics.

Mr. Chen received the University of Maryland Future Faculty Fellowship in 2010 .

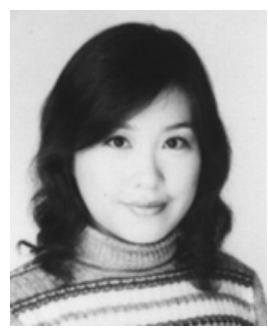

Beibei Wang (S'07) received the B.S. degree in electrical engineering (with highest honors) from the University of Science and Technology of China, Hefei, China, in 2004, and the Ph.D. degree in electrical engineering from the University of Maryland, College Park, in 2009

She is currently a Research Associate with the Department of Electrical and Computer Engineering, University of Maryland. Her current research interests include dynamic spectrum allocation and management in cognitive radio systems, wireless communications and networking, game theory, wireless multimedia, and network security.

Dr. Wang was the recipient of the Graduate School Fellowship, the Future Faculty Fellowship, and the Dean's Doctoral Research Award from the University of Maryland. 


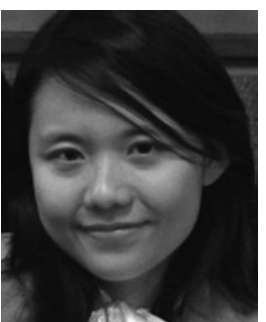

W. Sabrina Lin (M'06) received the B.S. and M.S. degrees from National Taiwan University, Taipei City, Taiwan, in 2002 and 2004, respectively, and the Ph.D. degree from the University of Maryland, College Park, in 2009, all in electrical engineering.

She is currently with the Department of Electrical and Computer Engineering, University of Maryland. Her current research interests include the area of information security and forensics, multimedia signal processing, and multimedia social network analysis.

Dr. Lin received the University of Maryland Future

Faculty Fellowship in 2007.

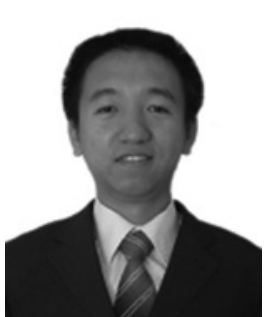

Yongle Wu (S'08) received the B.S. (with highest honors) and M.S. degrees in electronic engineering from Tsinghua University, Beijing, China, in 2003 and 2006, respectively. He is currently working toward the Ph.D. degree from the Department of Electrical and Computer Engineering, University of Maryland, College Park.

His current research interests include the areas of wireless communications and networks, including cognitive radio techniques, dynamic spectrum access, and network security.

Mr. Wu received the Graduate School Fellowship from the University of Maryland in 2006, and the Future Faculty Fellowship from the A. James Clark School of Engineering, University of Maryland, in 2009.

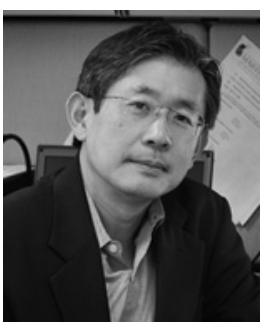

K. J. Ray Liu (F’03) was a Distinguished ScholarTeacher with the University of Maryland, College Park, in 2007. He is an Associate Chair of the Graduate Studies and Research of the Department of Electrical and Computer Engineering and leads the Maryland Signals and Information Group where he conducts research encompassing broad aspects of wireless communications and networking, information forensics and security, multimedia signal processing, and biomedical engineering. His recent books include Cognitive Radio Networking and Security: A Game Theoretical View (Cambridge University Press, 2010), Cooperative Communications and Networking (Cambridge University Press, 2008), Resource Allocation for Wireless Networks: Basics, Techniques, and Applications (Cambridge University Press, 2008), Ultra-Wideband Communication Systems: The Multiband OFDM Approach (IEEE-Wiley, 2007), NetworkAware Security for Group Communications (Springer, 2007), Multimedia Fingerprinting Forensics for Traitor Tracing (Hindawi, 2005), and Handbook on Array Processing and Sensor Networks (IEEE-Wiley, 2009).

$\mathrm{He}$ is the recipient of numerous honors and awards, including the IEEE Signal Processing Society Technical Achievement Award and Distinguished Lecturer Award. He received various teaching and research recognitions from the University of Maryland, including the University-Level Invention of the Year Award, and the Poole and Kent Senior Faculty Teaching Award and Outstanding Faculty Research Award, both from the A. James Clark School of Engineering. He is a Fellow of AAAS. He is the President-Elect and was the Vice President of Publications of the IEEE Signal Processing Society. $\mathrm{He}$ was the Editor-in-Chief of the IEEE SIGNAL PROCESSING MAGAZINE and the Founding Editor-in-Chief of the EURASIP Journal on Advances in Signal Processing. 